\title{
The extracellular HDAC6 ZnF UBP domain modulates the actin network and post-translational modifications of Tau
}

\author{
Abhishek Ankur Balmik ${ }^{1,2+}$, Shweta Kishor Sonawane ${ }^{1,2 \dagger}$ and Subashchandrabose Chinnathambi ${ }^{1,2^{*}}$ (0)
}

\begin{abstract}
Background: Microtubule-associated protein Tau undergoes aggregation in Alzheimer's disease (AD) and a group of other related diseases collectively known as Tauopathies. In AD, Tau forms aggregates, which are deposited intracellularly as neurofibrillary tangles. Histone deacetylase-6 (HDAC6) plays an important role in aggresome formation, where it recruits polyubiquitinated aggregates to the motor protein dynein.

Methods: Here, we have studied the effects of HDAC6 ZnF UBP on Tau phosphorylation, ApoE localization, GSK-3 $\beta$ regulation and cytoskeletal organization in neuronal cells by immunocytochemical analysis. This analysis reveals that the cell exposure to the UBP-type zinc finger domain of HDAC6 (HDAC6 ZnF UBP) can modulate Tau phosphorylation and actin cytoskeleton organization.

Results: HDAC6 ZnF UBP treatment to cells did not affect their viability and resulted in enhanced neurite extension and formation of structures similar to podosomes, lamellipodia and podonuts suggesting the role of this domain in actin re-organization. Also, HDAC6 ZnF UBP treatment caused increase in nuclear localization of ApoE and tubulin localization in microtubule organizing centre (MTOC). Therefore, our studies suggest the regulatory role of this domain in different aspects of neurodegenerative diseases. Upon HDAC6 ZnF UBP treatment, inactive phosphoryl-

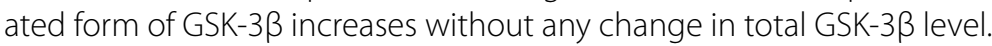

Conclusions: HDAC6 ZnF UBP was found to be involved in cytoskeletal re-organization by modulating actin dynamics and tubulin localization. Overall, our study suggests that ZnF domain of HDAC6 performs various regulatory functions apart from its classical function in aggresome formation in protein misfolding diseases.

Keywords: HDAC6, Tau, Phosphorylation, Tauopathies, Microtubule, Actin, Podosomes, Podonut, Actin, Cytoskeleton, Neurodegeneration

\footnotetext{
*Correspondence: s.chinnathambi@ncl.res.in

${ }^{\dagger}$ Abhishek Ankur Balmik and Shweta Kishor Sonawane have equally contributed

${ }^{1}$ Neurobiology Group, Division of Biochemical Sciences, CSIR-National Chemical Laboratory (CSIR-NCL), Dr. Homi Bhabha Road, Pune 411008, India

Full list of author information is available at the end of the article
}

\section{Background}

The misfolded proteins are either refolded by chaperones or targeted for degradation via ubiquitin proteasomal system (UPS) [1-4]. Upon failure or disruption of the UPS, aggregates are directed towards the formation of aggresomes, which serve as a cytoprotective response upon UPS failure [4-7]. HDAC6 is a class II histone deacetylase primarily present in cytoplasm. This protein is involved in regulation of various cellular functions. It consists of two catalytic deacetylase domains (histone deacetylase 1 and 2, residues 87-404 and 482-800 original author(s) and the source, provide a link to the Creative Commons licence, and indicate if changes were made. The images or other third party material in this article are included in the article's Creative Commons licence, unless indicated otherwise in a credit line to the material. If material is not included in the article's Creative Commons licence and your intended use is not permitted by statutory regulation or exceeds the permitted use, you will need to obtain permission directly from the copyright holder. To view a copy of this licence, visit http://creativecommons.org/licenses/by/4.0/. The Creative Commons Public Domain Dedication waiver (http://creativeco mmons.org/publicdomain/zero/1.0/) applies to the data made available in this article, unless otherwise stated in a credit line to the data. 
respectively) and a unique ZnF UBP (Zinc finger ubiquitin binding protein) domain (residues 1131-1192), which sets HDAC6 apart from other HDACs [8-10]. One of the major functions of HDAC6 is recruitment of polyubiquitinated protein aggregates to Dynein/Dynactin complex to sequester them to microtubule organizing centre (MTOC) in the perinuclear region for aggresome formation, thereby facilitating their clearance by autophagy [8-10]. Another important function of HDAC6 is the promotion of autophagosome-lysosome fusion and completion of autophagy [11-13]. The function of HDAC6 in both UPS and autophagy indicate its role as a possible link between the two mechanisms [11-13]. The impairment of UPS function acts as a cue for the activation of compensatory mechanisms for clearance of protein aggregates. It has been studied in the Drosophilla model of spinobulbar muscular atrophy, where expression of HDAC6 has been found to effectively cause rescue from UPS impairment-induced neurodegeneration by triggering the autophagic clearance of protein aggregates [12]. In another study, HeLa cells transfected with PolyQ Huntingtin showed the formation of intracellular protein aggregates, which require HDAC6 for autophagic clearance after inhibition of proteasomal system [14]. Overall, the function of HDAC6 with respect to protein aggregate clearance and autophagy induction serves as an important protective mechanism. In line with these considerations, expression levels of HDAC6 increases sharply in protein misfolding diseases [15]. The functions of $\mathrm{ZnF}$ UBP domain of HDAC6 are less understood as compared to the function of its catalytic domains. The possible role of HDAC6 ZnF UBP in directly modulating the aggregation propensity and stability of Tau has been recently reported [16].

Phosphorylation is a well-studied PTM of Tau responsible for its conversion to pathological form during $A D$ pathogenesis [17-19]. Alzheimer`s disease is associated with upregulation of cellular kinases, such as GSK-3 $\beta$ and CDK5 [20]. GSK-3 $\beta$ is one of the major kinases involved in Tauopathies [21]. Tau is phosphorylated by GSK-3 $\beta$ on both primed (after pre-phosphorylation) and unprimed sites and such phosphorylation affects the ability of Tau to bind and stabilize microtubules [22, 23]. Many serine and threonine residues within the repeat region of Tau have been mapped as potential phosphorylation sites by proline-directed kinases [24]. On the other hand, protein phosphatases, such as PP1 and PP2A, are known to be downregulated in $\mathrm{AD}$, failing to reverse the effect of hyperphosphorylation [25-27]. Therefore, there is an imbalance between the kinase and phosphatase function in neurons under pre-pathological conditions. We have studied the effects of HDAC6 ZnF UBP domain on phospho-Tau epitopes-pT181 and AT8, as well as on the level of total and downregulated form of GSK-3 $\beta$. HDAC6 ZnF UBP was found to lower both phospho-epitopes and promoted GSK- $3 \beta$ downregulation.

Another important aspect of neurodegenerative diseases involve mis-functioning of cytoskeletal elements. Microtubule network and actin organization were found to be distorted in neurodegeneration leading to impaired cellular trafficking and distortion of other associated functions $[28,29]$. Actin organization is crucial for synaptic signalling in neurons, where it is involved in formation of dendritic spines for neurotransmission. Impaired actin assembly and depolymerisation lead to the loss of dendritic spines, ultimately causing neuronal death [30, 31]. HDAC6 is a key protein in the regulation of both actin and microtubule organization through its deacetylase activity [32]. HDAC6 acts on cortactin and mediates its association with $\mathrm{F}$-actin (fibrous actin) to facilitate cell motility [33]. In present study, we studied the role of HDAC6 ZnF UBP domain in modulation of cytoskeletal assembly independent of its catalytic domains. The localization of ApoE either in cytoplasm or nucleus serves as an indicator of neuronal health. Nuclear localization of ApoE is associated with increased neuronal survival. Enhanced ApoE nuclear localization was observed in neurons in the presence of HDAC6 ZnF UBP, suggesting that HDAC6 ZnF UBP can promote ApoE nuclear localization in HSP90 and nucleolin mediated manner.

Overall, in this study, HDAC6 ZnF UBP domain was found to affect actin and tubulin organization, Tau phosphorylation, and localization of ApoE in neuronal cells. Enhanced formation of podosome and lamellipodia-like structures was found, when neuronal cells were exposed to HDAC6 ZnF UBP domain, suggesting direct role of this domain in actin organization. HDAC6 $\mathrm{ZnF}$ UBP treatment also resulted in enhanced tubulin localization in MTOC, indicating the possible role of this domain in tubulin polymerization events as well. Our findings suggest that HDAC6 ZnF UBP acts as a direct modulator of Tau phosphorylation and cytoskeletal dynamics.

\section{Materials and methods Chemicals and reagents}

Luria-Bertani broth (Himedia); Ampicillin, $\mathrm{NaCl}$, Phenylmethylsulfonylfluoride (PMSF), $\mathrm{MgCl}_{2}$, APS, DMSO, Ethanol (Mol Bio grade), Isopropanol (Mol Bio grade) were purchased from MP biomedicals; IPTG and Dithiothreitol (DTT) from Calbiochem; MES, BES, SDSfrom Sigma; EGTA, Protease inhibitor cocktail, Tris base, $40 \%$ Acrylamide, TEMED from Invitrogen. For cell culture studies, Dulbecco modified eagle's media (DMEM), Fetal bovine Serum (FBS), Horse serum, Phosphate buffer saline (PBS, cell biology grade), Trypsin-EDTA, Penicillin-streptomycin, Pierce ${ }^{\mathrm{TM}}$ LDH Cytotoxicity Assay Kit 
(Thermo, cat no 88953), RIPA buffer were also purchased from Invitrogen. MTT reagent, Okadaic acid and TritonX-100 were purchased from Sigma. The coverslip of $18 \mathrm{~mm}$ was purchased from Bluestar for immunofluorescence. In immunofluorescence and western blot study we used the following antibodies: Beta-actin (Thermofisher cat no. MA515739) Beta Tubulin (BT7R) (Thermofisher, cat no MA516308) and total Tau antibody K9JA (Dako, cat no A0024), pT181 (Invitrogen, cat no 701530) AT8 (Thermo fisher, cat no MN1020), GSK-3 $\beta$ (Thermo fisher, cat no MA5-15109), Phospho-GSK-3 $\beta$ (Ser9) (Thermo fisher, cat no MA5-14873), anti-ApoE (Sigma, cat no. SAB2701946), anti-mouse secondary antibody conjugated with Alexa Fluor-488 (Invitrogen, cat no A-11001), Goat anti-Rabbit IgG $(\mathrm{H}+\mathrm{L})$ Cross-Adsorbed Secondary Antibody with Alexa Fluor 555 (A-21428), Rabbit antiGoat IgG $(\mathrm{H}+\mathrm{L})$ Cross-Adsorbed Secondary Antibody with Alexa Fluor 594 (A27016) and DAPI (Invitrogen).

\section{Protein expression and purification}

Full length Tau (hTau40wt) in pT7C were transformed and expressed in BL21* cells while HDAC6 ZnF UBP in pET28a-LIC was transformed and expressed in BL21 Codon plus RIL cells. Full-length Tau and repeat domain Tau were purified in two steps using cation exchange chromatography and size-exclusion chromatography. The cells expressing these proteins after transformation were scaled up and harvested. The cells were lysed by homogenization at 15,000 KPSI. The lysate was supplied with $0.5 \mathrm{M} \mathrm{NaCl}$ and $5 \mathrm{mM}$ DTT and kept at $90{ }^{\circ} \mathrm{C}$ for $20 \mathrm{~min}$ to denature all structured protein. The resulting sample was centrifuged at 40,000 rpm for $45 \mathrm{~min}$. The supernatant was kept for overnight dialysis in $20 \mathrm{mM}$ MES pH 6.8. The dialyzed sample was centrifuged again at 40,000 rpm for $45 \mathrm{~min}$ and the supernatant was filtered and loaded onto Sepharose fast flow (SPFF) column pre-equilibrated with $20 \mathrm{mM} \mathrm{MES} \mathrm{pH} \mathrm{6.8,} 50 \mathrm{mM} \mathrm{NaCl}$. Elution was carried out using $20 \mathrm{mM}$ MES pH 6.8, $1 \mathrm{M}$ $\mathrm{NaCl}$. The fractions collected from cation exchange chromatography containing Tau protein were pooled, concentrated and subjected to size-exclusion chromatography using 1X PBS, 2 mM DTT in Superdex 75 Hi-load 16/600 column [34, 35]. Purification of HDAC6 ZnF UBP was carried out by Ni-NTA affinity chromatography using $50 \mathrm{mM}$ Tris-Cl pH 8.0 with $20 \mathrm{mM}$ Imidazole for wash and $1000 \mathrm{mM}$ imidazole for elution. The sample was dialyzed overnight in $50 \mathrm{mM}$ Tris-Cl pH 8.0, $100 \mathrm{mM} \mathrm{NaCl}$, $2.5 \%$ glycerol to remove imidazole followed by size-exclusion chromatography using Superdex 75 Hi-load 16/600 column [36].

\section{Cell viability by MTT assay}

The effective concentration of HDAC6 for subsequent treatments was determined by studying the concentration dependent toxicity studies by MTT assay. $10^{4}$ neuro2a cells (ATCC CCL-131) were seeded in a 96 well culture plate in DMEM supplemented with $10 \%$ FBS and antibiotic penicillin-streptomycin for $24 \mathrm{~h}$ at $37^{\circ} \mathrm{C}$ $\mathrm{CO}_{2}$ incubator. The cells were treated with HDAC6 $(0-500 \mathrm{nM})$ in serum-starved media for $24 \mathrm{~h}$. MTT at a concentration of $0.5 \mathrm{mg} / \mathrm{mL}$ was added to the cells and incubated for $3 \mathrm{~h}$. The reduction of MTT by cellular enzymes forms formazon crystals, which were dissolved in DMSO, and the colour developed was quantified by reading at $570 \mathrm{~nm}$ in a TECAN Infinite $200 \mathrm{PRO}$ plate reader.

\section{LDH assay}

The effect of HDAC6 0-500 nM treatment on cell membrane integrity was studied by LDH (Lactate Dehydrogenase) assay. The disruption of cell membrane integrity leads to the leakage of LDH enzyme, which is quantified by an enzymatic reaction giving a colored end product. For performing LDH assay, the cells were incubated and treated as mentioned for MTT assay. After treatment with HDAC6, supernatant media was used and assay was performed according to manufacturer's protocol. In brief, $50 \mu \mathrm{L}$ of cell supernatant was incubated with $50 \mu \mathrm{L}$ of the reaction mixture provided for $30 \mathrm{~min}$ at room temperature. $50 \mu \mathrm{L}$ of stop solution was added to each well and the colour developed was measured at $490 \mathrm{~nm}$ and background subtraction at $680 \mathrm{~nm}$ was done.

\section{Caspase 3/7 activity assay}

In order to study the effect of HDAC6 on inducing apoptotic cell death, activity of executioner caspase 3 was determined by EnzChek ${ }^{\mathrm{TM}}$ Caspase-3 Assay Kit. 10,000 cells/well cells were seeded in a 12 well culture plate for $24 \mathrm{~h}$ and further treated with HDAC6 (0-500 $\mathrm{nM}$ ) for $24 \mathrm{~h}$ in serum-starved media. Caspase activity was performed as per manufacturer's protocol. The cells were lysed with provided lysis buffer in freeze-thaw cycles. The cell debris was centrifuged out and supernatant was incubated with fluorescent substrate (DEVD-Rhodamine). The fluorescence was quantified at (Ex/Em) 496 $1520 \mathrm{~nm}$ at different time intervals in TECAN Infinite 200 PRO plate reader.

\section{Cell culture, immunofluorescence and quantitative analysis}

Neuro2a cells from passage number 10-20 were cultured in advanced DMEM supplemented with penstrep-glutamine, anti-mycotic and 10\% FBS. For immunofluorescence studies $5 \times 10^{4}$ cells were seeded on a glass coverslip 


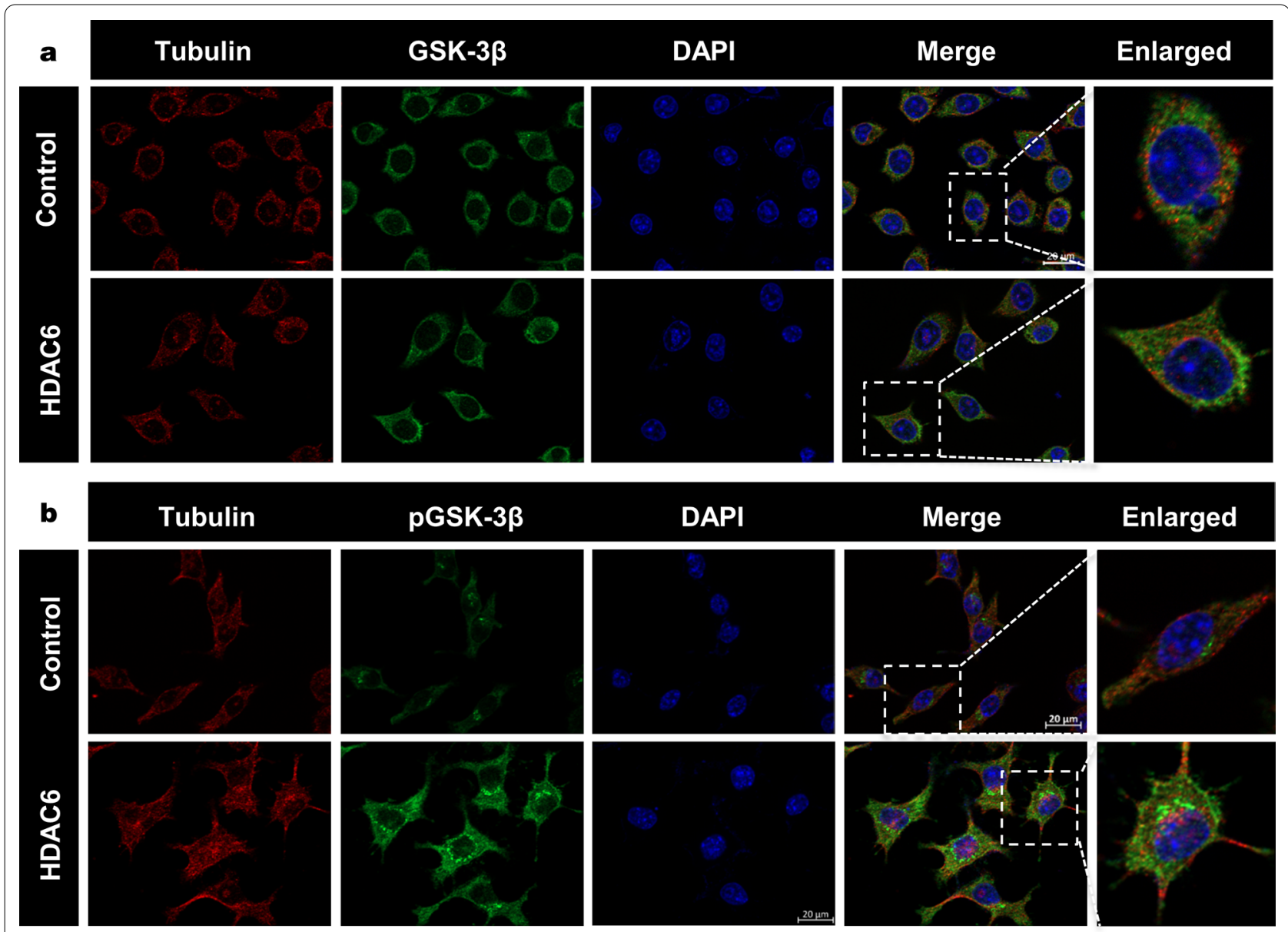

C

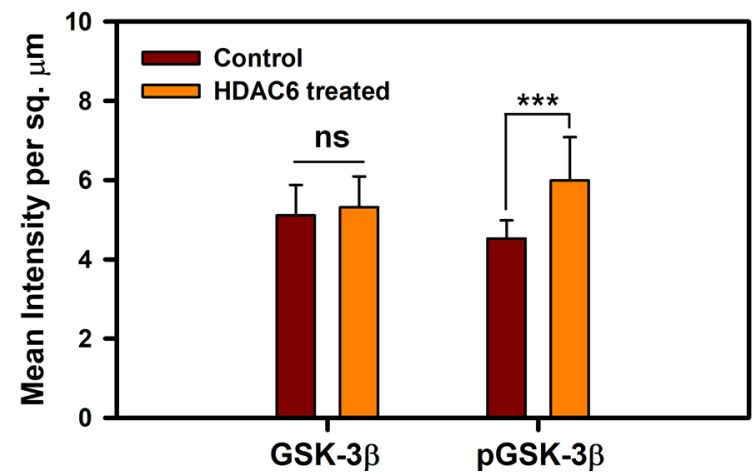

Fig. 1 Downregulation of GSK-3 $\beta$ activity by HDAC6. a Neuro2a mapped for total GSK-3 $\beta$ shows their unaltered levels upon HDAC6 ZnF UBP. $\mathbf{b}$ Inhibitory phosphorylation of GSK-3 $\beta$ at Ser9 increases upon HDAC6 ZnF UBP treatment. The enlarged image shows the elevated level of $p G S K-3 \beta$ compared to neuro2a cell control. c Quantification of mean fluorescence intensity for GSK-3ß in cell control and HDAC6 ZnF UBP treated cells showed non-significant difference while the levels of pGSK-3 $\beta$ were significantly increased upon HDAC6 ZnF UBP treatment in comparison to cell control

(Bluestar) in a 12 well culture plate. Cells were given desired treatment in serum starved media (0.5\% FBS) for $24 \mathrm{~h}$ including groups involving $25 \mathrm{nM}$ Okadaic acid (OA) treatment. After incubation period, cells were washed with PBS and fixed with $4 \%$ paraformaldehyde. Further cells were washed with PBS thrice and permiabilized using $0.2 \%$ Triton X-100. Cells were blocked with $2 \%$ horse serum and incubated with primary antibodies 


\section{(See figure on next page.)}

Fig. 2 Inhibition of Tau phosphorylation by HDAC6. a HDAC6 treatment for $24 \mathrm{~h}$ along with OA shows inhibition of Tau phosphorylation at pT181 epitope whereas OA treatment shows increased phosphorylation at PT181. b Phosphorylation at AT8 epitope is also reduced in presence of HDAC and OA as compared to OA alone. The enlarged image of neuro2a treated with OA alone and OA along with HDAC6 ZnF UBP shows marked difference in the level of phosphorylation at pT181 and AT8 epitopes. $\mathbf{c}$, $\mathbf{d}$ Mean fluorescence intensity for AT8 immunostaining of untreated neuro2a (CC), okadaic acid treatment (OA), HDAC6 ZnF UBP treatment (HDAC6) and HDAC6 ZnF UBP treatment along with okadaic acid. Okadaic acid treatment resulted in increased level of pT181 and AT8 phospho-epitopes. HDAC6 ZnF UBP treatment with OA reduced the level of both phospho-epitopes. Quantification data was analyzed by one way ANOVA followed by Tukey's HSD test. Significant at mean difference $>$ T (Tukey's criterion) and $a=0.05$. Groups with the same letters are not significantly different. The value of T is calculated as 0.365 for pT181 and 1.341 for AT8 quantification

in a moist chamber at $4{ }^{\circ} \mathrm{C}$ overnight. Next day, cells were washed thrice with $1 \mathrm{X}$ PBS and incubated with alexa fluor labeled secondary antibodies for $1 \mathrm{~h}$ at $37^{\circ} \mathrm{C}$. The unbound secondary antibody was washed off with three washes of PBS and counterstained with DAPI. The coverslips were mounted in $80 \%$ glycerol and observed under 63X oil immersion lens in Axio Observer 7.0 Apotome 2.0 (Zeiss) microscope. The quantification of immunofluorescence intensity was carried out by Zen 2.3 software and mean fluorescence intensity per unit area were determined for various treatment groups in respective number of fields (n).

\section{Statistical analysis}

Two-tailed unpaired student $\mathrm{t}$-test was used to determine the significance for experiments involving comparison of two groups (n.s. - non-significant, * indicates $P \leq 0.05$, ** indicates $P \leq 0.01$, *** indicates $P \leq 0.001$ ). One way ANOVA was conducted for the levels of phosphoepitopes (pT181 and AT8) to compare different treatment groups. Tukey's HSD (Honest significant difference) test was performed to compare the significance within groups (Significant at Tukey's HSD $p<0.05$ ). All the experiments were performed in triplicates and analyzed by Sigmaplot 10.0 (Systat software).

\section{Results}

\section{HDAC6 ZnF UBP is non-toxic and non-apoptotic}

Neuronal cells were exposed to HDAC6 ZnF UBP exogenously and effects of this exposure on different cellular functions were analyzed (Additional file 1: Fig. 1A). The cell viability analysis carried out by MTT assay showed no noticeable decrease in viability, which was maintained at $80 \%$ even in the presence of highest HDAC6 ZnF UBP concentration of $500 \mathrm{nM}$ (Additional file 1: Fig. 1B). Similarly, LDH assay was utilized to check the effect of HDAC6 on membrane integrity in terms of LDH release. Neuronal cells showed intact membrane when treated with HDAC6 ZnF UBP in 20-500 nM concentration range (Additional file 1: Fig. 1C). Neuronal cells were treated with a range of concentrations of HDAC6 $\mathrm{ZnF}$ UBP (20-500 nM), and its internalization was monitored by immunostaining for HDAC6 and anti-His-tag (Additional file 1: Fig. 2). To further confirm the non-toxic nature of HDAC6 ZnF UBP for neuronal cells, we used caspase-3 assay to study the induction of apoptosis (if any) associated with treatment of cells with HDAC6 ZnF UBP. Under stressful conditions, cells undergo apoptosis mediated by endoproteases called caspases [37, 38]. Caspase-3 is an executioner caspase responsible for DNA fragmentation and degradation of cytoplasmic proteins [39-41]. The caspase- 3 assay showed basal level of activity in all the treated and control samples. No difference was observed between HDAC6 ZnF UBP treated and untreated control samples in terms of cell viability, suggesting that HDAC6 ZnF UBP treatment did not induce apoptosis in neuro2a cells (Additional file 1: Fig. 1D). Therefore, these studies on cell viability and morphology inferred that HDAC6 ZnF UBP treatment did not show cytotoxic effects on neuro2a cells. Hence, in subsequent experiments, cells were treated with a moderate concentration of $50 \mathrm{nM}$ HDAC6 ZnF UBP, unless stated otherwise.

\section{HDAC6 ZnF UBP enhances $p G S K-3 \beta$ levels}

We mapped GSK-3 $\beta$ and pGSK-3 $\beta$ (pSer9) levels by immunofluorescence in neuronal cells in basal conditions and upon HDAC6 ZnF UBP treatment (No. of fields selected for quantification of GSK-3 $\beta$ and pGSK-3 $\beta$ (pSer9), $n=6$ ). There was no marked difference in GSK-3 $\beta$ levels in HDAC6-treated and untreated cells (Fig. 1a). However, immunofluorescence analysis of HDAC6 ZnF UBP-treated cells showed significant increase in pGSK-3 $\beta$ levels (Fig. 1b). GSK-3 $\beta$ function in cells is regulated mainly by the inhibitory phosphorylation at Ser9 or Ser21 residues. The kinase activity of GSK-3 $\beta$ is reduced with phosphorylation at Ser9, as it affects the binding of primed substrates to GSK-3 $\beta$. Mean fluorescence intensity of cell control and HDAC6 ZnF UBP treated cells showed non-significant changes in GSK-3 $\beta$ levels but pGSK-3 $\beta$ levels were found to be increased in HDAC6 ZnF UBP treated cells (Fig. 1c). The increase in pGSK-3 $\beta$ in neuronal cells signifies reduced GSK-3 $\beta$ activity on primed Tau substrate [42]. 

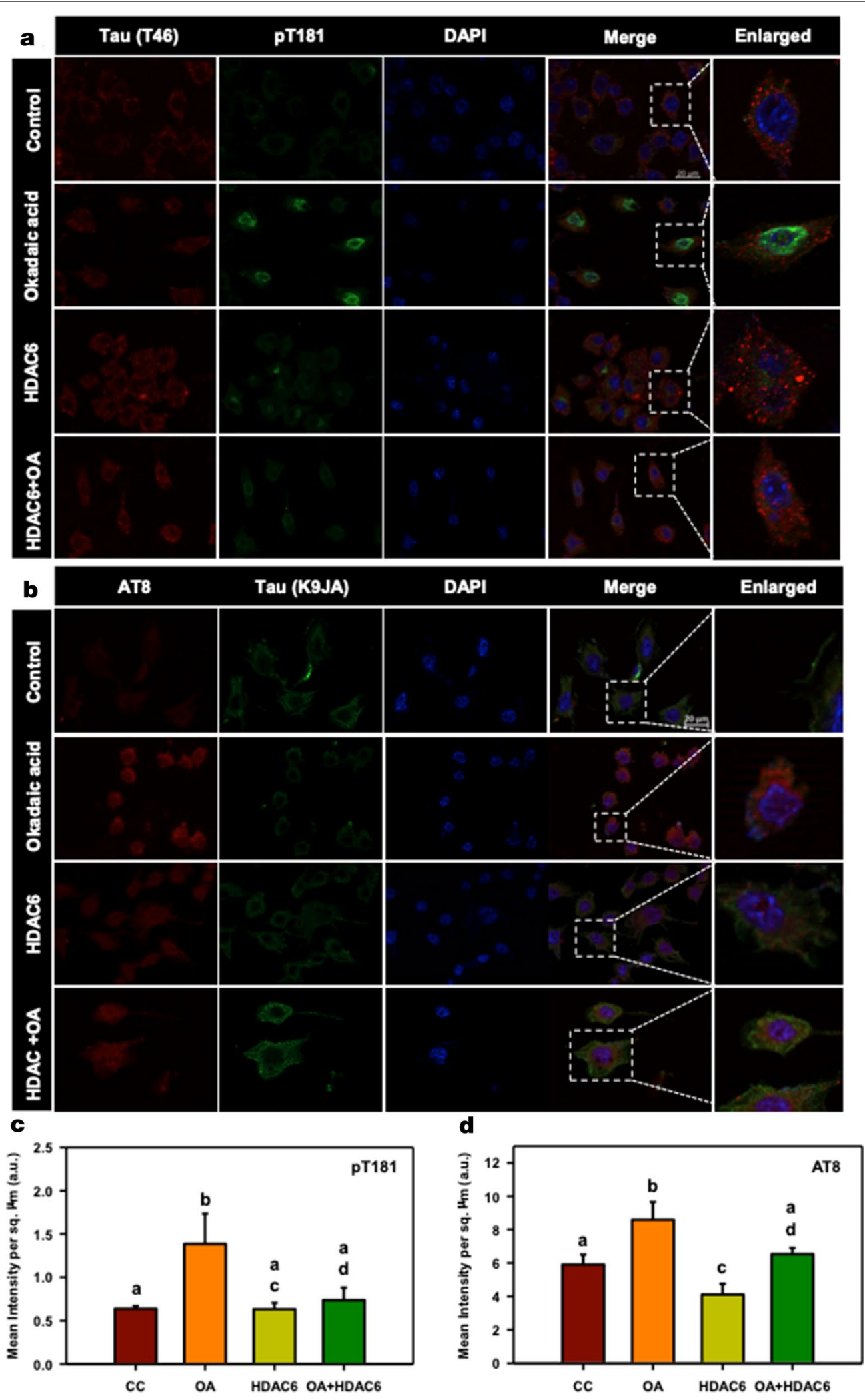


\section{(See figure on next page.)}

Fig. 3 HDAC6 as a modulator of actin dynamics. HDAC6 deacetylase activity is known to modulate actin dynamics. The effect on actin dynamics was studied after exposure to HDAC6 ZnF UBP domain. HDAC6 ZnF UBP treatment to neuro2a resulted in enhancement of neurite extensions which are mapped by F-actin staining. a HDAC6 ZnF UBP treatment for $24 \mathrm{~h}$ increases the neuronal extensions as compared to untreated control cells when the extensions are mapped by actin immunostaining. The Tau levels remain unaltered in both the groups. $\mathbf{b}$ The orthogonal projection shows increase in the number of neurites in HDAC6 ZnF UBP treated cells. c Greyscale images for untreated neuro2a cells mapped for F-actin showed small extensions in the growth cones. $\mathbf{d}$ In HDAC6 ZnF UBP treated cells, F-actin containing longer extensions were observed. Growth cones were observed to be concentrated in membrane extensions and filopodia-like structures

The HDAC6 ZnF UBP treatment of cells resulted in the increased levels of pGSK-3 $\beta$ (Ser9), which in turn enables reduced Tau phosphorylation. The increased levels of pGSK- $3 \beta$ suggested down-regulated GSK- $3 \beta$ activity under the influence of HDAC6 ZnF UBP.

\section{HDAC6 ZnF UBP reduces Tau phosphorylation in neuronal cells}

Aberrant Tau phosphorylation is one of the key events in AD pathogenesis. Tau phosphorylation is required for its function in microtubule interaction and stabilization. Under pathological conditions, Tau becomes hyperphosphorylated due to imbalance in kinase and phosphatase levels or activity $[17-19,43]$ Okadaic acid (OA) was used as an inducer of hyperphosphorylation, as it inhibits protein phosphatase $2 \mathrm{~A}$ (PP2A), thereby increasing the overall phosphorylation level [44]. Untreated control cells and HDAC6 ZnF UBP-treated cells alone showed basal levels of phospho-Tau at epitopes pT181 and pS202/ T205 (AT8). The levels of pT181 were increased in OA treated cells. Cells supplemented with HDAC6 ZnF UBP with OA showed lower levels of phospho Tau at T181 as compared to the positive control (Fig. 2a). Tau phosphorylated at pT181 was dominantly seen in the nucleus, especially in positive control (enlarged images). Similar results were observed for phospho Tau epitope AT8. The enlarged images clearly show increased levels of phospho-AT8 in positive control as compared to OA + HDAC6 ZnF UBP treatment (Fig. 2b). pT181 and AT8 are two of the crucial epitopes of pathological Tau in AD. HDAC6 ZnF UBP treatment lowered down the level of these two phospho-epitopes, suggesting the possible role of this domain in modulating Tau phosphorylation. The level of both phospho-tau epitopes (pT181 and AT8) were quantified by their immunofluorescence levels in different experimental groups (No. of fields selected for quantification of pT181 and AT8, n=5). Untreated cells (CC) showed no significant difference from HDAC6treated or OA + HDAC6-treated cells in the levels of pT181and AT8 immunostaining. OA treatment resulted in increased pT181and AT8 levels, which were found to be reduced with HDAC6 ZnF UBP treatment (Fig. 2c, d). Quantification data were analyzed by one-way ANOVA followed by Tukey's HSD test (significant at $\alpha=0.05$ ).
The statistical significance was calculated with respect to Tukey's criterion $(\mathrm{T})$. The groups with same letters show no significant difference.

\section{HDAC6 ZnF UBP modulates actin dynamics}

In neuronal cells, actin dynamics is important for cellto-cell communication through regulating neurite extension. HDAC6 is known to modulate actin organization through its association with and deacetylase activity on cortactin and arp $2 / 3$ complex $[33,45,46]$. The function of HDAC6 ZnF UBP domain in actin dynamics is not understood as of yet. Our observations showed that HDAC6 ZnF UBP treatment can lead to an increase in neuritic extensions in neuro2a cells. We mapped HDAC6 ZnF UBP-treated cells with FITC-Phalloidin to observe the changes in F-actin cytoskeleton. We studied actin organization along with Tau and observed that HDAC6 ZnF UBP treatment had no effect on Tau levels but the actin cytoskeleton changed significantly (Fig. 3a). Further, to check for the changes in Tau localization on treatment, we mapped actin with Tau. The control cells showed a normal F-actin cytoskeleton in the extensions, but HDAC6-treated cells showed presence of actin along with Tau in these extensions (Fig. 3b). We also observed Tau-actin co-localization in neuritic extensions indicating the interplay of microtubule and actin dynamics in formation of cell extensions (Additional file 1: Fig. S3A, B). Greyscale images for actin immunostaining shows localization in neurite extensions and growth cones in filopodia-like structures (Fig. 3c, d).

\section{Enhancement of podosome-like structures by HDAC6 ZnF UBP}

Morphological changes in neuronal cells were observed when treated with HDAC6 ZnF UBP (Additional file 1: Fig. 4). HDAC6 ZnF UBP-treated cells showed increased membrane ruffles and podosome-like structures. Neuro2a cells were incubated with $50 \mathrm{nM}$ HDAC6 ZnF UBP on $18 \mathrm{~mm}$ coverslips for $24 \mathrm{~h}$ prior to immunostaining preparation. Immunostaining showed localization of actin in the membrane ruffles in HDAC6 $\mathrm{ZnF}$ UBPtreated cells. We observed minimal neuritic extensions in untreated neuro2a as compared to treated cells (Fig. 4a). Actin localization was observed mostly along the cell 


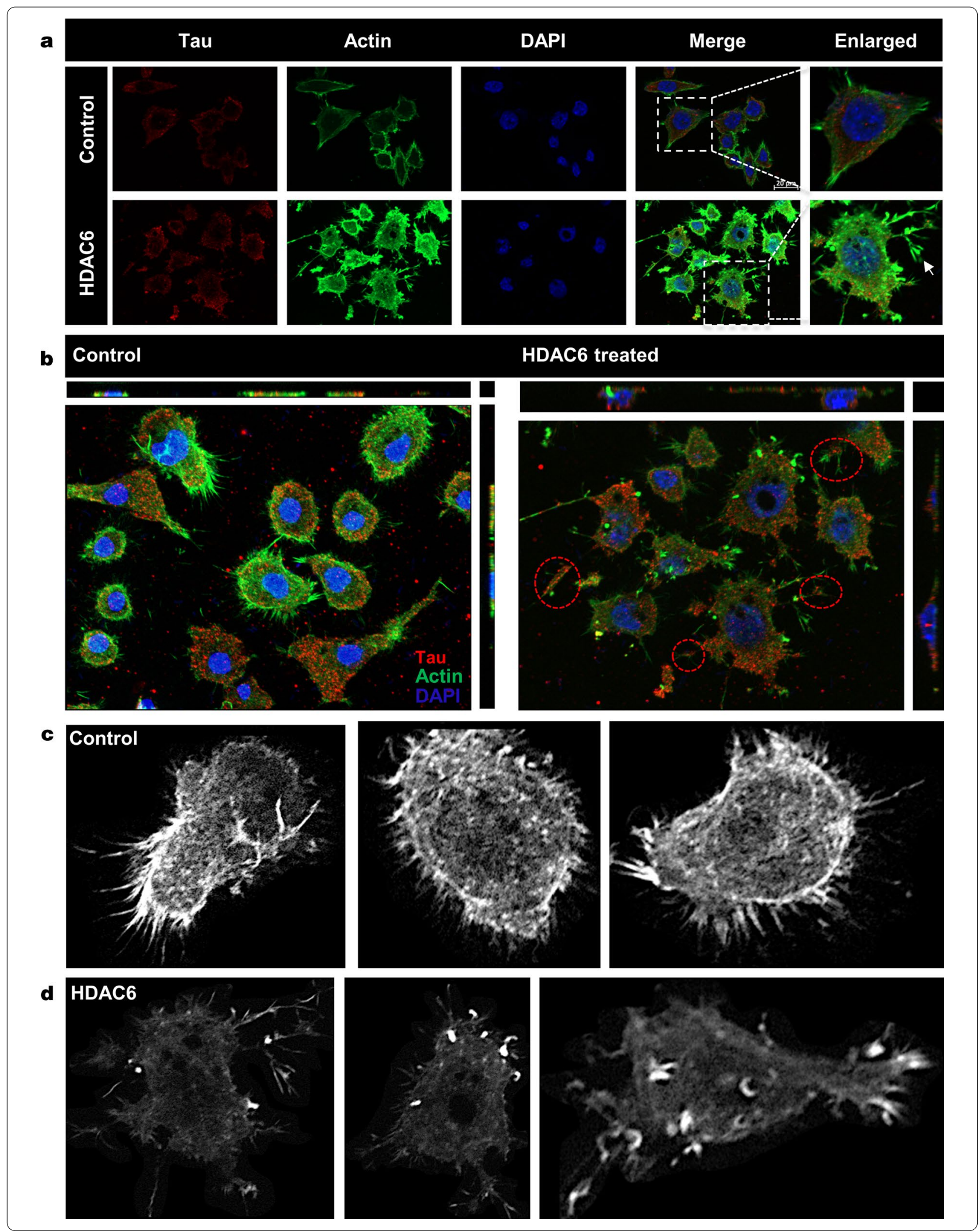




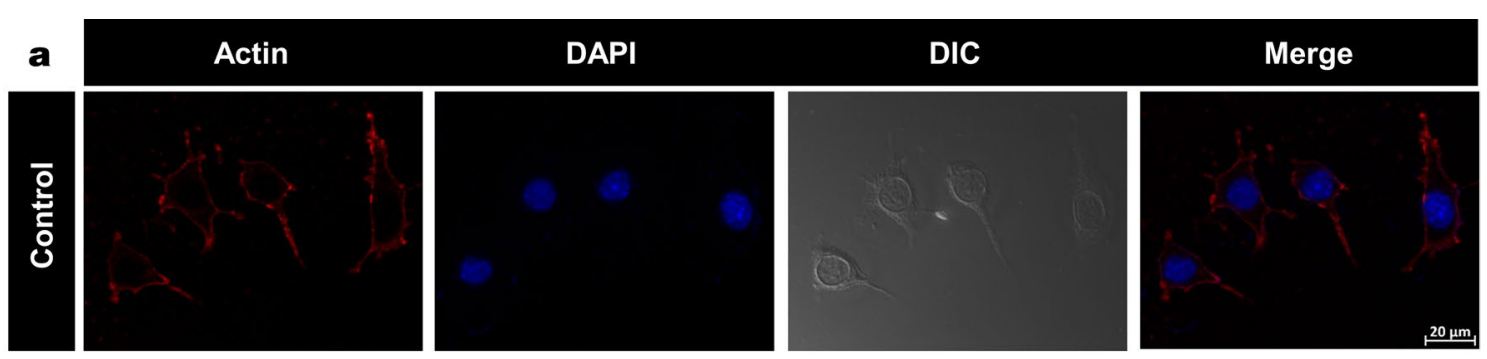

\section{b}
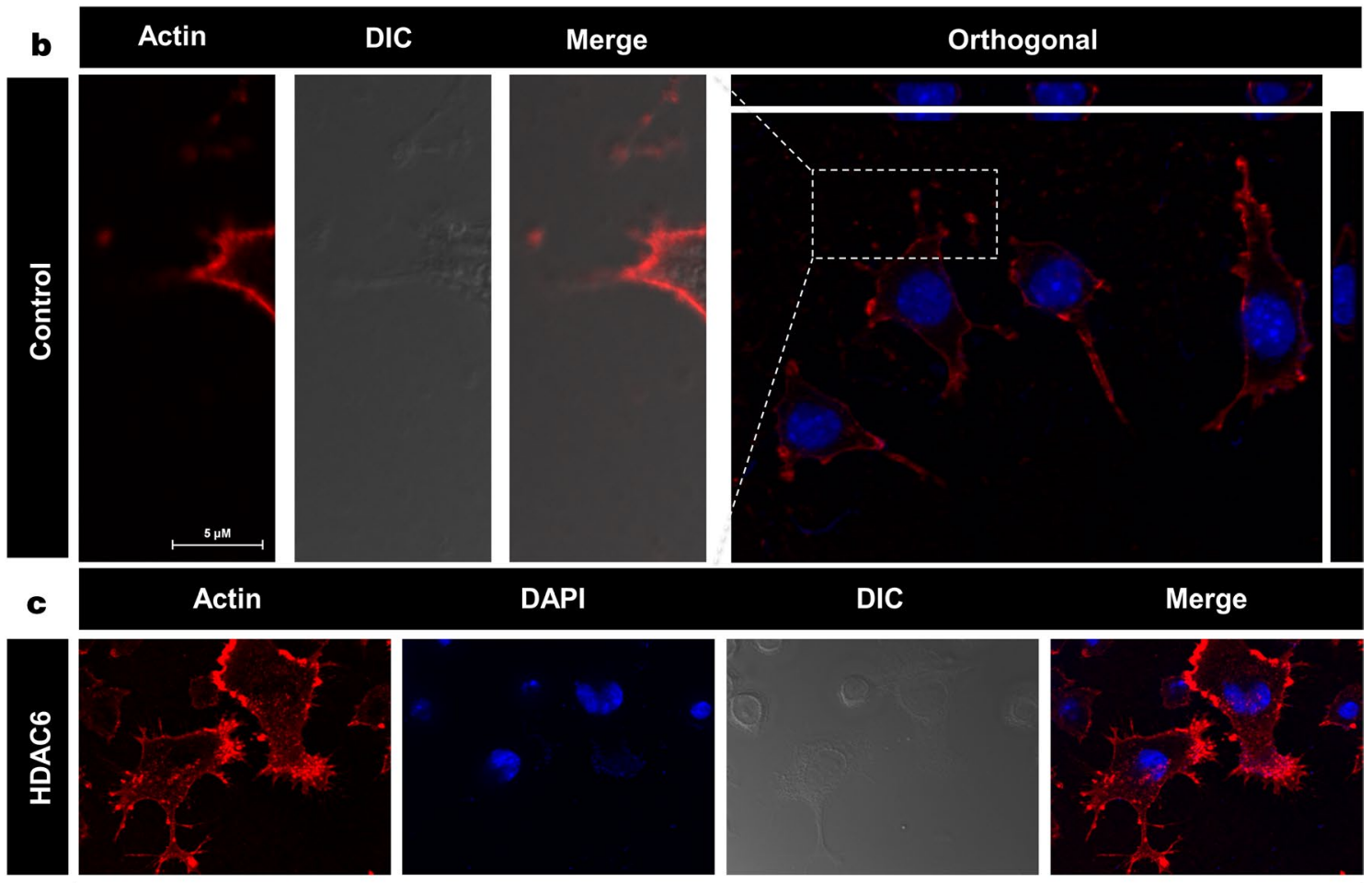

DAPI

DIC

Merge
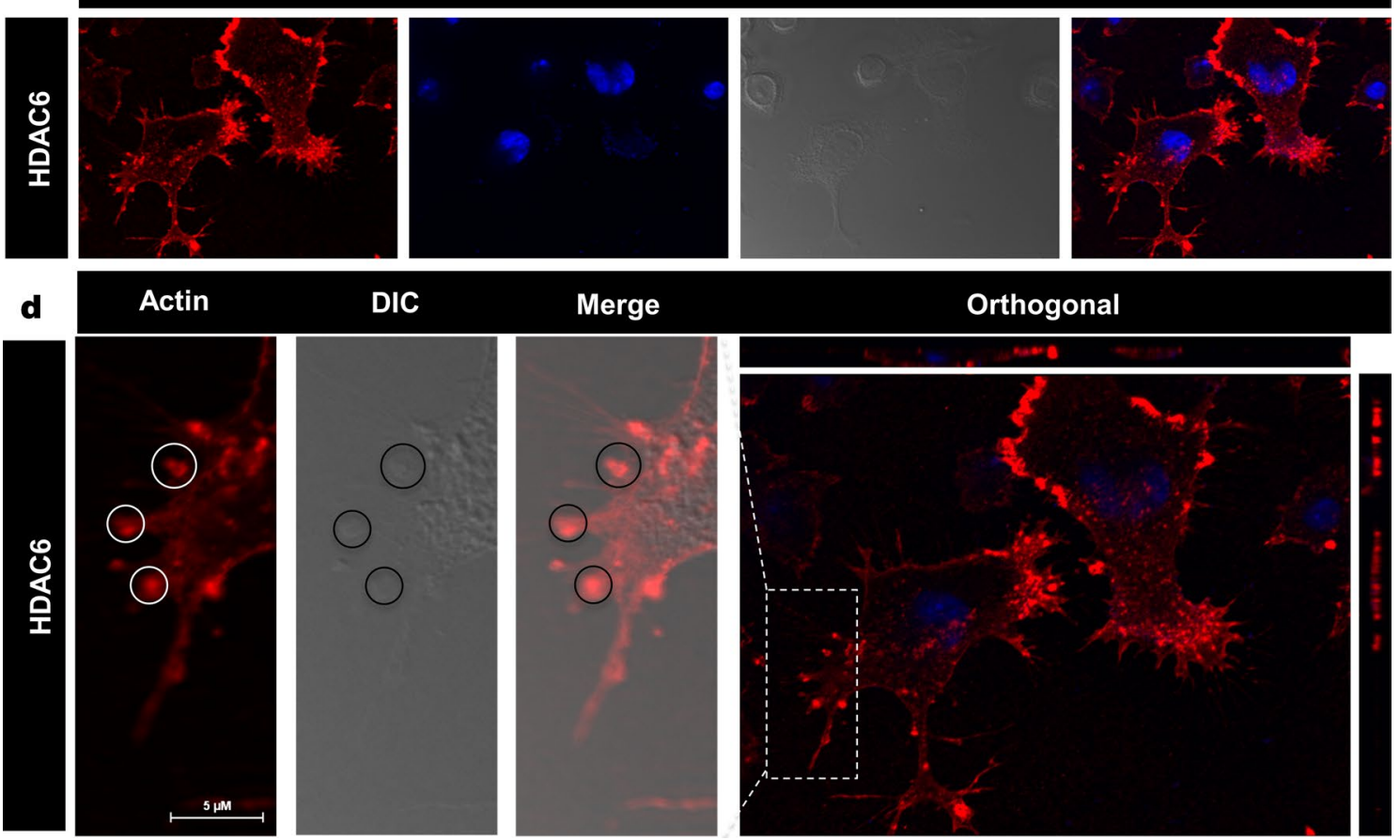

d

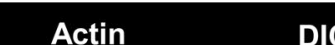

\section{Merge}

Orthogonal
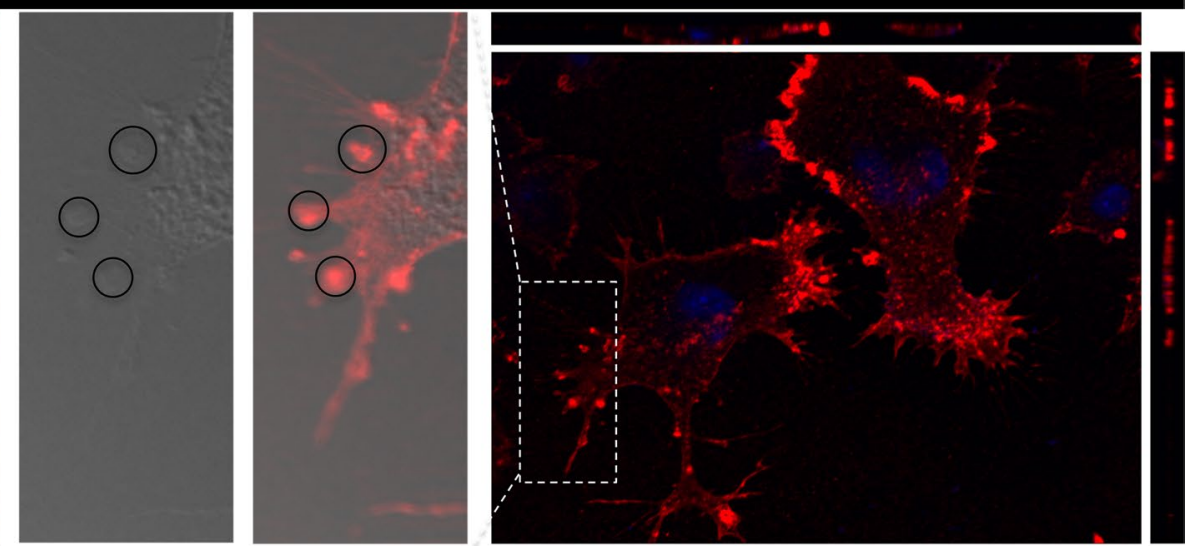

Fig. 4 Enhancement of podosome formation by HDAC6. a Podosomes marked by actin rich structures along plasma membrane, were observed in neuro2a cells at a minimal level. b Enhancement in podosome like structures observed in HDAC6 ZnF UBP treated cells. Orthogonal projection images showed marked difference in membrane morphology and actin concentrated in membrane ruffles and podosome like structures in HDAC6 ZnF UBP treated cells. $\mathbf{c ~ H D A C 6}$ is found to be present in neuritic extensions along with actin suggesting its role in regulation of neurite extensions. This is not seen in case of untreated cells as indicated in enlarged images for both groups. $\mathbf{d}$ In HDAC6 ZnF UBP treated cells, F-actin containing longer extensions were observed. Growth cones were observed to be concentrated in membrane extensions and filopodia-like structures 


\begin{abstract}
(See figure on next page.)
Fig. 5 Podosome, lamellipodia and podonut-like structure induced by HDAC6 ZnF UBP. Neuro2a cells exposed to HDAC6 ZnF UBP exhibit a variety of actin rich structures characteristics of migratory or invading cells. $\mathbf{a}-\mathbf{c}$ ) Untreated neuro2a cells showed actin uniformly distributed along the periphery with small neuritic extensions while $\mathbf{d}$, e HDAC6 ZnF UBP treated cells showed longer extensions and membranes ruffles resembling podosomes involved in cell migration. $\mathbf{f}, \mathbf{g}$ Invadopodia and lamellipodia-like structures (encircled) were also observed, which are found in phagocytic cells. $\mathbf{h}$, i Most of the treated cells were observed to consist of assemblance of podosomes (encircled) and podosome clusters called podonuts (white arrow) rich in actin. $\mathbf{j}$ The percentage of cells with or without podosomes/neurite extensions was calculated for control and HDAC6 ZnF UBP treatments groups. There were $<20 \%$ cells in control with podosome-like structures while $>80 \%$ cells in exhibited podosome-like structures upon HDAC6 ZnF UBP treatments. In cell control, 65-70\% cells had neurite extensions, which were calculated to be 80-85\% in HDAC6 ZnF UBP treated cells. (P + and P- represent cells with and without podosomes respectively. $\mathrm{N}+$ and $\mathrm{N}$ - represent cells with or without neuritic extensions respectively.) $\mathbf{k}$ The overall neurite extensions in neuro2a cells were counted in untreated and HDAC6 treated group in $\beta$-actin immunostained cells. The number of extensions in HDAC6 treated cells were found to be greatly enhanced compared to untreated group. I The podosomes formed after HDAC6 ZnF UBP treatment were quantified by counting the podosome crown structures in both untreated and treated groups. HDAC6 ZnF UBP treated cells showed more podosome clusters signifying its actin modulating effect. Statistical significance determined by two-tailed unpaired t-test. (n.s. - non-significant, ${ }^{*}$ indicates $P \leq 0.05$, ${ }^{* *}$ indicates $P \leq 0.01,{ }^{* * *}$ indicates $P \leq 0.001$ )
\end{abstract}

periphery in untreated neuro2a, while it was focused in the neurite extensions and protruding podosome-like structure in HDAC6 ZnF UBP-treated cells (Fig. 4b). Orthogonal sections were taken to clearly visualize F-actin driven extensions and podosome formation. The initial orthogonal sections showed numerous extensions, and actin localized in the tip of extensions in HDAC6 ZnF UBP-treated cells as compared to control (Fig. 4c, d). Involvement of podosomes in cell adhesion and migration is an important attribute of invasive cells. Neuro2a, being a neuroblastoma cell line, can form podosomes for cell migration and attachment. In contrast to untreated control neuronal cells (Fig. 5a-c), HDAC6 ZnF UBPtreated cells showed enhanced cell extensions and actinrich structures similar to podosomes formed in migrating and invading cells. HDAC6-treated cells showed a variety of actin-based membrane protrusions which can be morphologically classified as filopodia or lamellipodia, podosomes and podonuts (Fig. $5 \mathrm{~d}-\mathrm{i}$ ). The neuritic extensions and podosomes were quantified in untreated control cells (CC) and HDAC6-treated cells. The neurites and podosomes were counted manually in random multiple fields for untreated control and HDAC6 treated group (Total no. of fields, $\mathrm{n}=6$ ). Overall, $<20 \%$ podosomes containing cells were observed in cell control while $>80 \%$ of HDAC6 ZnF UBP treated cells exhibited podosomes-like structures. Similarly, 65-70\% cells with neurite extensions were found in cell control while $80-85 \%$ cells had enhanced neurite extensions in HDAC6 treated cells (Fig. 5j). HDAC6 ZnF UBP treated cells showed significantly enhanced neurite extensions and podosomes compared to untreated cells implicating its effect on actin dynamics and re-organization (Fig. 5k, l). Statistical significance of control and HDAC6 treated cells was analyzed by two-tailed unpaired student $t$-test.

\section{HDAC6 ZnF UBP enhances ApoE nuclear localization}

In normal physiological conditions, ApoE is localized in cytosol. ApoE nuclear localization has been reported in ovarian cancer cells, where it leads to better survival possibly through gene regulation [47]. We treated neuro2a cells with $50 \mathrm{nM}$ of HDAC6 ZnF UBP domain to observe its effect on distribution and localization of ApoE in cells. It was observed that upon HDAC6 ZnF UBP treatment, there was an increase in nuclear localization of ApoE (Fig. 6a). The localization of ApoE was quantified by analyzing the intensity of ApoE immunofluorescence in nucleus and cytoplasm of untreated and HDAC6 ZnF UBP-treated cells (No. of fields selected for quantification, $\mathrm{n}=10$ and the statistical analysis carried out by using two-tailed unpaired student t-test.). The ApoE level in cytoplasm of both untreated and HDAC6 treated cells showed no change while it was significantly increased in the nucleus of HDAC6 ZnF UBP-treated cells (Fig. 6b). The enhanced nuclear localization of ApoE may indicate an improved neuronal health [48].

\section{Enhanced Tubulin localization to MTOC with HDAC6 ZnF UBP treatment}

HDAC6 is a known interacting partner of tubulin that regulates microtubule structure and function through deacetylation. In a previous study, it was found that HDAC6 knockdown in cells along with HDAC6 inhibitor tubacin treatment does not affect microtubule growth velocity [49]. This implies that the effects of HDAC6 that are independent of its deacetylase activity may also exist. HDAC6 ZnF UBP treatment was given to neuro2a cells to observe the effect of this domain on microtubule network. Such HDAC6 ZnF UBP treatment of neuro2a cells increased tubulin localization around nucleus in MTOC (Fig. 6c). When neuro2a cells were mapped for actin and tubulin, untreated cells showed more axonal localization of tubulin along with actin, while HDAC6 treated 


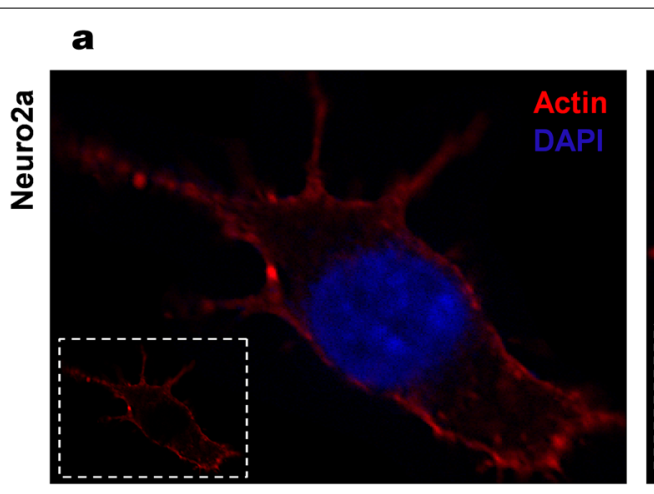

d

0
咊
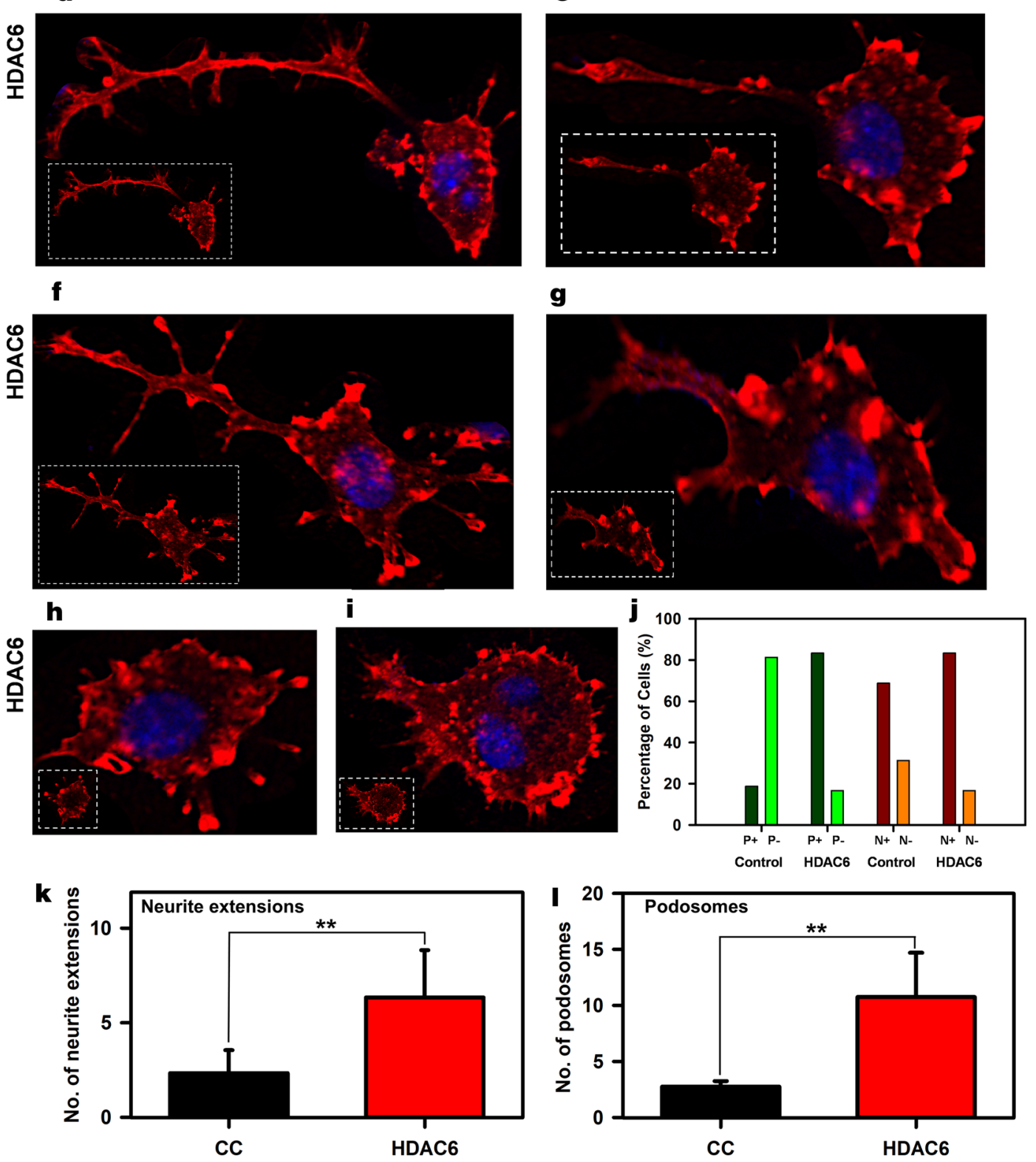
(See figure on next page.)

Fig. 6 Modulation of ApoE and Tubulin localization by HDAC6. a Immunofluorescence mapping for ApoE and Tau upon HDAC6 treatment in neuro2a cells shows increased nuclear localization of ApoE. b ApoE intensity in nucleus and cytoplasm was quantified for untreated and HDAC6 $\mathrm{ZnF}$ UBP treated neuro2a immunostained with anti-ApoE antibody. There was no significant difference in cytoplasmic level of ApoE in both groups while nuclear ApoE fraction was notably increased in HDAC6 ZnF UBP treated cells. c Neuro2a treated with HDAC6 ZnF UBP shows localization of tubulin focused over nuclear periphery compared to untreated neuro2a where tubulin is evenly distributed in cytoplasm. Orthogonal projection image shows tubulin localization in nuclear periphery in HDAC6 ZnF UBP treated neuro2a cells. Statistical significance determined by two-tailed unpaired t-test. (n.s. - non-significant, ${ }^{*}$ indicates $P \leq 0.05,{ }^{* *}$ indicates $P \leq 0.01,{ }^{* * *}$ indicates $P \leq 0.001$ )

cells showed tubulin predominantly in MTOC (Additional file 1: Fig. 3a, b). The re-orientation of MTOC is a complex process, which occurs in a dynein, cdc42, and dynactin-dependent manner [50, 51]. Our results suggest the possible role of HDAC6 ZnF UBP domain in microtubule organization. However, the molecular mechanisms and functions of MTOC re-orientation are poorly understood.

\section{Discussion}

Histone deactylases are known to be the enzymes that act on histones and regulates epigenetic function in nucleus. However, many non-histone substrates of HDACs in cytoplasm have been established [10]. Among the classes of histones deacetylase, class IIb deacetylase HDAC6 holds a distinctive position, being the major cytoplasmic deacetylase having two active catalytic domains [10, 52]. HDAC6 exhibit domain-wise functions, which may or may not be dependent on each other. HDAC6 plays a major role in cell proliferation, cell migration, misfolded protein degradation and stress response through its deacetylase function or interacting with various other proteins [53]. HDAC6 levels are found to be elevated in $\mathrm{AD}$ patients following increase in the aggregate burden in neuronal cells [15]. This suggests the possibility of involvement of this protein and its HDAC6 $\mathrm{ZnF}$ UBP domain in tackling aggregates in a deacetylase independent manner. Neuro2a cells treated with various concentrations of HDAC6 ZnF UBP to assess their viability upon treatment using MTT assay and LDH assay. The presence of HDAC6 ZnF UBP showed no toxicity on Neuro2a. pT181 and AT8 (pS202/pT205) are two pathological phosphorylation events of Tau implicated in AD [20]. Okadaic acid treatment increases the level of these two epitopes by inhibiting PP2a activity. Treatment of cells with HDAC6 ZnF UBP decreased the levels of both pT181 and AT8. The effect possibly arose from replenishing the PP2a activity upon inhibition by OA rather than via inhibition of Tau phosphorylation [54] Class II HDACs-HDAC1, HDAC6, and HDAC10, can form molecular complexes with phosphatases (PP1 or PP2a). However, HDAC6 was shown to interact only with the catalytic subunit of PP1 to form a complex retaining both phosphatase and deacetylase catalytic activity [55].
The binding of HDAC6 to PP1 was mapped to the second catalytic domain and C-terminal domain of HDAC6, which corresponds to ZnF UBP domain [54]. The level of GSK-3 $\beta$ in its phosphorylated and non-phosphorylated form determines its activity as a kinase. GSK- $3 \beta$ is known to associate with HDAC6 to counteract its function to induce LPS-tolerance in astrocytes [56]. GSK-3 $\beta$ is a versatile protein kinase with more than hundred substrates [42]. GSK-3 $\beta$ acts on Tau in two different manners; i.e., on pre-phosphorylated primed Tau and unprimed Tau [23]. In order for GSK-3 $\beta$ to work on primed substrate, the $\mathrm{N}$-terminal primed substrate binding domain provides binding-site for primed substrates, thereby up-regulating the kinase activity of GSK-3 $\beta$. Phosphorylation of GSK- $3 \beta$ at Serine 9 is the regulatory mechanism to downregulate activity of this kinase [22]. N-terminal domain with pSer9 acts as a pseudosubstrate for the primed substrate binding-domain of GSK-3 $\beta$ restricting the binding of other primed substrates, such as Tau [57]. Modulation of GSK-3 $\beta$ by HDAC6 ZnF UBP may involve regulation of Akt via PP1. PP1 dephosphorylates Akt, which is a negative regulator of GSK-3 $\beta$ in its phosphorylated state [58].HDAC6 ZnF UBP was found to increase the level of pSer9 phosphorylation on GSK-3 $\beta$ indicative of down-regulated GSK-3 $\beta$ activity with HDAC6 treatment in neuro2a cells.

Cellular functions, such as cell growth, morphogenesis, migration, intracellular transport, and attachment require co-ordinated operation of cytoskeletal networks of actin and microtubules. Both Tau and HDAC6 are known to be involved in the regulation of both these cytoskeletal networks [59]. Although HDAC6 exerts its regulatory function through catalytic domain, the role of HDAC6 ZnF UBP domain in this aspect needs to be explored. We studied the effects of HDAC6 $\mathrm{ZnF}$ UBP, on cytoskeletal organization and found the ability of this domain to restructure cytoskeletal network. HDAC6 ZnF UBP treatment resulted in enhancement of neuritic extensions. HDAC6 is an important modulator of cell migration and structure by associating with actin and tubulin polymerization machinery of cells [33, 49]. Podosomes are actin-based structures involved in the remodeling of extracellular matrix $(\mathrm{ECM})$ and cell migration, while podonut consists of a cluster of 


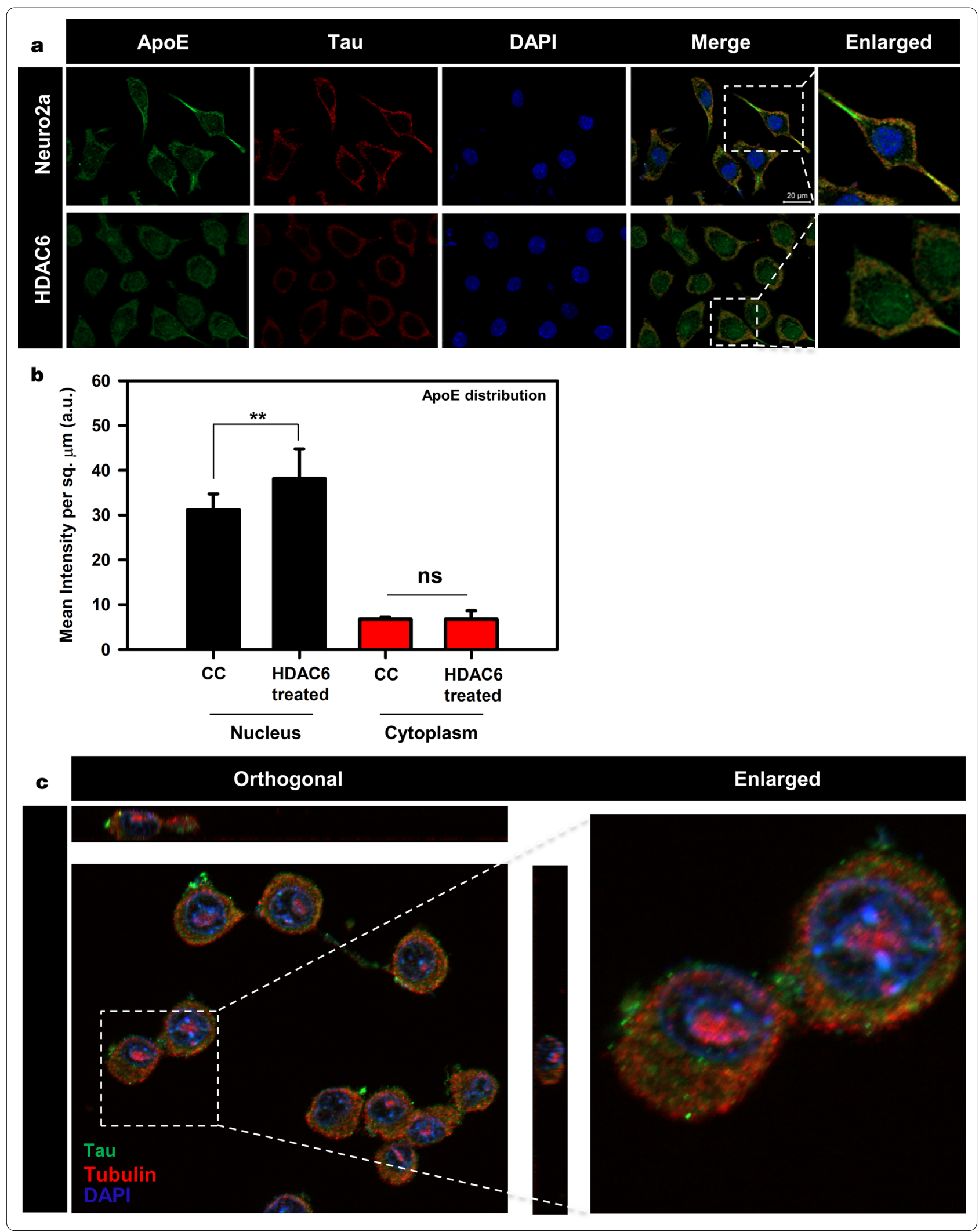




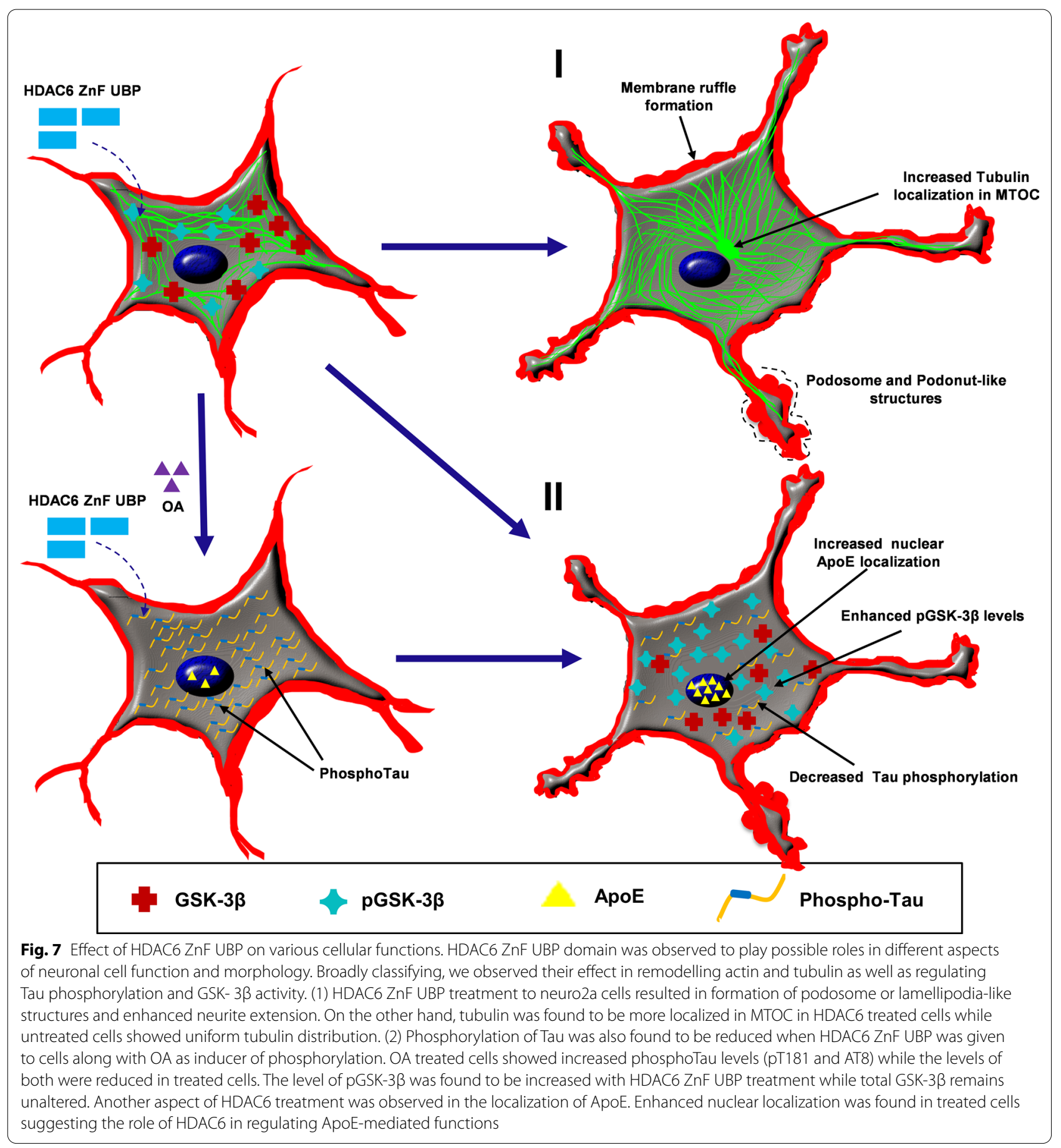

podosomes interacting with ECM [60]. Podosome consists of an actin-rich core surrounded by actin regulatory molecules, such as cortactin and arp $2 / 3$, as well as cell adhesion molecules, such as Talin and Vinculin [61]. We observed the co-localization of actin and HDAC6 in neurite extensions and formation of podosomes structures involved in cell migration in HDAC6 $\mathrm{ZnF}$ UBP treated cells. Podosomes are membranes invaginations formed in cells like macrophages, dendritic, smooth muscle, invasive cancer cells and in post-synaptic apparatus [62]. The observed results revealed the ability of HDAC6 ZnF UBP to enhance cell migration and neurite extensions by promoting actin assembly along cell periphery. The role of HDAC6 in cytoskeletal organization has been 
studied extensively. HDAC6 has been reported to play regulatory role in chemotactic movement and migration of lymphocytes in deacetylase independent manner [63]. HDAC6 also regulate membrane ruffle formation in HSP90 and Rac1 mediated mechanism and found to be localized in membrane ruffles along with actin [64]. In this aspect, the role of HDAC6 ZnF UBP in promoting neuritic extensions by actin remodelling can prove to be a novel aspect of HDAC6 in neuroprotection. As previously observed, HDAC6 ZnF UBP-treated cells showed more neuritic extensions and dense actin cytoskeleton. In previous studies, Tau and actin were shown to co-localize in neuritic extensions, where they facilitated growth cone transition [65]. HDAC6 ZnF UBP treatment also leads to enhanced localization of tubulin around nucleus signifying the possible structural and pathological aspect of HDAC6 ZnF UBP. ApoE is the major apolipoprotein of central nervous system, produced primarily by astrocytes. ApoE can get translocated into nucleus through a nuclear targeting chaperone nucleolin [47]. ApoE contains a weak nuclear localizing sequence, which indicates that there must be other mechanisms involved in ApoE nuclear transport. Nucleolin is known to associate with apolipoproteins, facilitating their translocation [66]. HDAC6 may mediate ApoE translocation through HSP90 as it is known to stabilize nucleolin during mitosis [67]. However, the exact mechanism needs to be further elucidated.

The function of HDAC6 ZnF UBP domain is known with respect to aggregate clearance by mediating aggresome formation. However, current findings suggest that this domain may have other regulatory functions (Fig. 7). Overall, our studies suggest the role of HDAC6 ZnF UBP domain in modulating functions crucial to neuronal health that are independent of HDAC6 catalytic activity.

\section{Conclusions}

We studied the role of HDAC6 ZnF UBP domain in different aspects of AD pathology. Upon HDAC6 ZnF UBP treatment, inactive phosphorylated form of GSK-3 $\beta$ increases without any change in total GSK-3 $\beta$ level. Decreased level of Tau phospho-epitopes pT181 and AT8 with HDAC6 ZnF UBP treatment indicates that it has a regulatory role in Tau phosphorylation. Also, HDAC6 $\mathrm{ZnF}$ UBP was found to be involved in cytoskeletal reorganization by modulating actin dynamics and tubulin localization. Enhanced neuritic extension and formation of podosome-like structure were observed with HDAC6 ZnF UBP treatment and tubulin was more localized in MTOC suggesting its regulatory function. ApoE translocation in nucleus enhanced upon HDAC6 ZnF UBP treatment implying its possible role in ApoE pathological cascade in AD. Overall, our study suggests that the UBP ZnF domain of HDAC6 performs various regulatory functions apart from its classical function in aggresome formation in protein misfolding diseases.

\begin{abstract}
Abbreviations
AD: Alzheimer's disease; ApoE: Apolipoprotein E; ECM: Extracellular matrix; FTDP-17: Frontotemporal dementia and parkinsonism linked to chromosome 17; GSK-3ß: Glycogen synthase kinase-3beta; HDAC6: Histone deacetylase 6; HDAC6 ZnF UBP: HDAC6 zinc finger ubiquitin binding protein domain; HSP90: Heat shock protein 90; IDP: Intrinsically disorder protein; IDPR: Intrinsically disorder protein region; MTOC: Microtubule organizing centre; NFTs: Neurofibrillary tangles; OA: Okadaic acid; PP1: Protein phosphatase 1; PP2A: Protein phosphatase 2A; UPS: Ubiquitin proteasomal system.
\end{abstract}

\section{Supplementary Information}

The online version contains supplementary material available at https://doi. org/10.1186/s12964-021-00736-9.

Additional file 1: Supplementary figure 1. HDAC6 ZnF UBP treatment to neuro2a cells does not have a toxic effect. A) Amino acid sequence of human HDAC6 Zinc finger ubiquitin-binding domain. This domain is located on the C-terminal of HDAC6 and associates with polyubiquitinated protein aggregates to mediate the formation of aggresomes. B) MTT assay was carried out to determine the viability of neuro2a upon HDAC6 ZnF UBP treatment. Neuroblastoma cells treated with HDAC6 at different concentrations show minimum toxicity and maintain viability at $80 \%$ at highest concentration of $500 \mathrm{nM}$. C) LDH release assay determines the damage to the cell membrane upon exposure to test molecule. The membrane leakage assay (LDH assay) shows that HDAC6 does not disrupt the cell membrane and affect cell viability. D) Apoptosis rate of neuro2a cells upon HDAC6 ZnF UBP treatment was assayed using Caspase-3 assay. Caspase- 3 assay shows increased levels of caspase- 3 with successive time interval but do not differ from control samples. Statistical significance determined by two-tailed unpaired t-test. (n.s. - non-significant, ${ }^{*}$ indicates $P \leq 0.05,{ }^{* *}$ indicates $P \leq 0.01,{ }^{* * *}$ indicates $P \leq 0.001$ ). Supplementary figure 2. Internalization of HDAC6 ZnF UBP in neuro2a. Neuro2a cells treated with HDAC6 ZnF UBP (20-500 nM) were mapped by antibody against HDAC6 and anti His-tag antibody to determine the internalization of 14 KDa HDAC6 ZnF UBP domain in cells. Supplementary figure 3. Co-localization of actin and tubulin in neuro2a. A) Immunostaining for $\beta$-actin and tubulin was performed to examine their localization in HDAC6 ZnF UBP treated neuro2a cells. Actin and tubulin co-localizes in the neuronal extensions in both treated and untreated cells. However, tubulin was found to be localized more in MTOC in case of HDAC6 treatment. B) Orthogonal projection images for actin and tubulin shows both actin and tubulin in untreated control cells while differential localization of tubulin in HDAC6 treated cells. Supplementary figure 4. DIC for neurite extensions in HDAC6 ZnF UBP treated cells. HDAC6 ZnF UBP treated cells showed enhancement in actin regulated membrane extensions in the form of podosomes, lamellipodia and podonuts. DIC images for untreated and HDAC6 treated groups indicate marked difference in the pattern of their membrane extensions.

\section{Acknowledgements}

This project is supported in part by grants from the Department of Biotechnology from Neuroscience Task Force (Medical Biotechnology-Human Development \& Disease Biology (DBT-HDDB))-BT/PR/19562/MED/122/13/2016 and in-house CSIR-National Chemical Laboratory grant MLP029526. Abhishek Ankur Balmik acknowledges the Shyama Prasad Mukherjee fellowship (SPMF) from Council of Scientific Industrial Research (CSIR), India. SKS acknowledges Department of Biotechnology for fellowship. Tau constructs were kindly gifted by Prof. Roland Brandt from University of Osnabruck, Germany. HDAC6 
ZnF UBP construct was kindly gifted by Prof. Cheryl Aerosmith, University of Toronto, Canada.

\section{Authors' contributions}

$A B, S K S$, and $S C$ conducted most of the experiments, analyzed the results, and wrote the paper. SC conceived the idea for the project and wrote the paper. All authors read, edited and approved the final manuscript.

\section{Availability of data and materials}

Not applicable.

\section{Declarations}

\section{Ethics approval and consent to participate}

Not applicable.

\section{Consent for publication}

Not applicable.

\section{Competing interests}

The authors declare no competing financial interest.

\section{Author details}

${ }^{1}$ Neurobiology Group, Division of Biochemical Sciences, CSIR-National Chemical Laboratory (CSIR-NCL), Dr. Homi Bhabha Road, Pune 411008 , India. ${ }^{2}$ Academy of Scientific and Innovative Research (AcSIR), Ghaziabad 201002, India.

Received: 7 November 2020 Accepted: 29 March 2021

Published online: 01 May 2021

\section{References}

1. Amm I, Sommer T, Wolf DH. Protein quality control and elimination of protein waste: the role of the ubiquitin-proteasome system. Biochimica et Biophysica Acta Mol Cell Res. 2014;1843:182-96.

2. Pierre $S-R$, Vernace $V$, Wang Z, Figueiredo-Pereira ME. Assembly of protein aggregates in neurodegeneration: mechanisms linking the ubiquitin/ proteasome pathway and chaperones. In Madame Curie Bioscience Database [Internet]. Landes Bioscience; 2013.

3. Ciechanover A, Kwon YT. Degradation of misfolded proteins in neurodegenerative diseases: therapeutic targets and strategies. Exp Mol Med. 2015;47:e147.

4. Gorantla NV, Chinnathambi S. Tau protein squired by molecular chaperones during Alzheimer's disease. J Mol Neurosci. 2018;66:356-68.

5. Tai H-C, Schuman EM. Ubiquitin, the proteasome and protein degradation in neuronal function and dysfunction. Nat Rev Neurosci. 2008;9:826

6. Casadei N, Pöhler A-M, Tomás-Zapico C, Torres-Peraza J, Schwedhelm I, Witz A, Zamolo I, De Heer R, Spruijt B, Noldus LP. Overexpression of synphilin-1 promotes clearance of soluble and misfolded alpha-synuclein without restoring the motor phenotype in aged A30P transgenic mice. Hum Mol Genet. 2013;23:767-81.

7. Ardley HC, Scott GB, Rose SA, Tan NG, Robinson PA. UCH-L1 aggresome formation in response to proteasome impairment indicates a role in inclusion formation in Parkinson's disease. J Neurochem. 2004:90:379-91.

8. Simões-Pires C, Zwick V, Nurisso A, Schenker E, Carrupt P-A, Cuendet M. HDAC6 as a target for neurodegenerative diseases: what makes it different from the other HDACs? Mol Neurodegener. 2013;8:7.

9. Verdin E, Dequiedt F, Kasler HG. Class II histone deacetylases: versatile regulators. Trends Genet. 2003;19:286-93.

10. Yao Y-L, Yang W-M. Beyond histone and deacetylase: an overview of cytoplasmic histone deacetylases and their nonhistone substrates. BioMed Res Int 2010; 2011.

11. Pandey UB, Batlevi Y, Baehrecke EH, Taylor JP. HDAC6 at the intersection of autophagy, the ubiquitin-proteasome system, and neurodegeneration. Autophagy. 2007;3:643-5.

12. Pandey UB, Nie Z, Batlevi Y, McCray BA, Ritson GP, Nedelsky NB, Schwartz SL, DiProspero NA, Knight MA, Schuldiner O. HDAC6 rescues neurodegeneration and provides an essential link between autophagy and the UPS. Nature. 2007:447:860.
13. Hao R, Nanduri P, Rao Y, Panichelli RS, Ito A, Yoshida M, Yao T-P. Proteasomes activate aggresome disassembly and clearance by producing unanchored ubiquitin chains. Mol Cell. 2013;51:819-28.

14. Iwata A, Riley BE, Johnston JA, Kopito RR. HDAC6 and microtubules are required for autophagic degradation of aggregated huntingtin. J Biol Chem. 2005;280:40282-92.

15. Zhang L, Sheng S, Qin C. The role of HDAC6 in Alzheimer's disease. J Alzheimers Dis. 2013;33:283-95.

16. Balmik AA, Chidambaram H, Dangi A, Marelli UK, Chinnathambi S. HDAC6 $\mathrm{ZnF}$ UBP as the modifier of Tau structure and function. Biochemistry. 2020;59:4546-62.

17. Biernat J, Gustke N, Drewes G, Mandelkow E. Phosphorylation of Ser262 strongly reduces binding of tau to microtubules: distinction between PHF-like immunoreactivity and microtubule binding. Neuron. 1993;11:153-63.

18. Biernat J, Mandelkow E, Schröter C, Lichtenberg-Kraag B, Steiner B, Berling B, Meyer H, Mercken M, Vandermeeren A, Goedert M. The switch of tau protein to an Alzheimer-like state includes the phosphorylation of two serine-proline motifs upstream of the microtubule binding region. EMBO J. 1992;11:1593-7.

19. Drewes G, Ebneth A, Preuss U, Mandelkow E-M, Mandelkow E. MARK, a novel family of protein kinases that phosphorylate microtubule-associated proteins and trigger microtubule disruption. Cell. 1997;89:297-308.

20. Šimić G, Babić Leko M, Wray S, Harrington C, Delalle I, Jovanov-Milošević N, Bažadona D, Buée L, De Silva R, Di Giovanni G. Tau protein hyperphosphorylation and aggregation in Alzheimer's disease and other tauopathies, and possible neuroprotective strategies. Biomolecules. 2016;6:6

21. Lucas JJ, Hernández F, Gómez-Ramos P, Morán MA, Hen R, Avila J. Decreased nuclear $\beta$-catenin, tau hyperphosphorylation and neurodegeneration in GSK-3 $\beta$ conditional transgenic mice. EMBO J. 2001:20:27-39

22. Cho JH, Johnson GV. Primed phosphorylation of tau at Thr231 by glycogen synthase kinase $3 \beta$ (GSK3 $\beta$ ) plays a critical role in regulating tau's ability to bind and stabilize microtubules. J Neurochem. 2004;88:349-58.

23. Cho J-H, Johnson GV. Glycogen synthase kinase $3 \beta$ phosphorylates tau at both primed and unprimed sites differential impact on microtubule binding. J Biol Chem. 2003;278:187-93.

24. Šimić G, Diana A, Hof PR. Phosphorylation pattern of tau associated with distinct changes of the growth cone cytoskeleton. In: Guidance Cues in the Developing Brain. Springer; 2003. pp. 33-48.

25. Arendt T, Holzer M, Fruth R, Brückner M, Gärtner U. Paired helical filamentlike phosphorylation of tau, deposition of $\beta / A 4$-amyloid and memory impairment in rat induced by chronic inhibition of phosphatase 1 and 2A. Neuroscience. 1995;69:691-8.

26. Sontag E, Hladik C, Montgomery L, Luangpirom A, Mudrak I, Ogris $E$, White CL III. Downregulation of protein phosphatase $2 A$ carboxyl methylation and methyltransferase may contribute to Alzheimer disease pathogenesis. J Neuropathol Exp Neurol. 2004;63:1080-91.

27. Vintém APB, Henriques AG. e Silva OAdC, e Silva EFdC: PP1 inhibition by $A \beta$ peptide as a potential pathological mechanism in Alzheimer's disease. Neurotoxicol Teratol. 2009:31:85-8.

28. Li B, Chohan MO, Grundke-lqbal I, Iqbal K. Disruption of microtubule network by Alzheimer abnormally hyperphosphorylated tau. Acta Neuropathol. 2007;113:501-11.

29. Penzes P, VanLeeuwen J-E. Impaired regulation of synaptic actin cytoskeleton in Alzheimer's disease. Brain Res Rev. 2011;67:184-92.

30. Fiala JC, Spacek J, Harris KM. Dendritic spine pathology: cause or consequence of neurological disorders? Brain Res Rev. 2002;39:29-54.

31. Selkoe DJ. Alzheimer's disease is a synaptic failure. Science. 2002:298:789-91.

32. Boyault C, Sadoul K, Pabion M, Khochbin S. HDAC6, at the crossroads between cytoskeleton and cell signaling by acetylation and ubiquitination. Oncogene. 2007;26:5468.

33. Zhang $X$, Yuan Z, Zhang Y, Yong S, Salas-Burgos A, Koomen J, Olashaw N, Parsons JT, Yang X-J, Dent SR. HDAC6 modulates cell motility by altering the acetylation level of cortactin. Mol Cell. 2007;27:197-213.

34. Gorantla NV, Khandelwal P, Poddar P, Chinnathambi S. Global conformation of tau protein mapped by Raman spectroscopy. In: Tau Protein. Springer; 2017. pp. 21-31. 
35. Gorantla NV, Shkumatov AV, Chinnathambi S. Conformational dynamics of intracellular tau protein revealed by CD and SAXS. In: Tau Protein. Springer; 2017. pp. 3-20.

36. Ouyang H, Ali YO, Ravichandran M, Dong A, Qiu W, Mackenzie F, DhePaganon S, Arrowsmith $\mathrm{CH}$, Zhai RG. Protein aggregates are recruited to aggresome by histone deacetylase 6 via unanchored ubiquitin $C$ termini. J Biol Chem. 2012;287:2317-27.

37. Li J, Yuan J. Caspases in apoptosis and beyond. Oncogene. 2008;27:6194-206.

38. Harvey NL, Kumar S. The role of caspases in apoptosis. In: Apoptosis. Springer; 1998. pp. 107-128.

39. Damelio M, Cavallucci V, Cecconi F. Neuronal caspase-3 signaling: not only cell death. Cell Death Differ. 2010;17:1104-14.

40. Porter AG, Jänicke RU. Emerging roles of caspase-3 in apoptosis. Cell Death Differ. 1999;6:99-104.

41. Slee EA, Adrain C, Martin SJ. Executioner caspase-3,-6, and-7 perform distinct, non-redundant roles during the demolition phase of apoptosis. J Biol Chem. 2001;276:7320-6.

42. Beurel E, Grieco SF, Jope RS. Glycogen synthase kinase-3 (GSK3): regulation, actions, and diseases. Pharmacol Ther. 2015;148:114-31.

43. Avila J. Tau kinases and phosphatases. J Cell Mol Med. 2008;12:258-9.

44. Boban M, Leko MB, Miškić T, Hof PR, Šimić G. Human neuroblastoma SH-SY5Y cells treated with okadaic acid express phosphorylated high molecular weight tau-immunoreactive protein species. J Neurosci Methods; 2018.

45. Lee SH, Dominguez R. Regulation of actin cytoskeleton dynamics in cells. Mol Cells. 2010;29:311-25.

46. Oda T, Iwasa M, Aihara T, Maéda Y, Narita A. The nature of the globular-to fibrous-actin transition. Nature. 2009;457:441.

47. Kim WS, Elliott DA, Kockx M, Kritharides L, Rye K-A, Jans DA, Garner B. Analysis of apolipoprotein E nuclear localization using green fluorescent protein and biotinylation approaches. Biochem J. 2008;409:701-9.

48. Rohn TT, Moore ZD. Nuclear localization of apolipoprotein E4: a new trick for an old protein. Int J Neurol Neurother. 2017;4:2.

49. Zilberman Y, Ballestrem C, Carramusa L, Mazitschek R, Khochbin S, Bershadsky A. Regulation of microtubule dynamics by inhibition of the tubulin deacetylase HDAC6. J Cell Sci. 2009;122:3531-41.

50. Sancho D, Vicente-Manzanares M, Mittelbrunn M, Montoya MC, GordónAlonso M, Serrador JM, Sánchez-Madrid F. Regulation of microtubuleorganizing center orientation and actomyosin cytoskeleton rearrangement during immune interactions. Immunol Rev. 2002;189:84-97.

51. Palazzo AF, Joseph HL, Chen Y-J, Dujardin DL, Alberts AS, Pfister KK, Vallee RB, Gundersen GG. Cdc42, dynein, and dynactin regulate MTOC reorientation independent of Rho-regulated microtubule stabilization. Curr Biol. 2001;11:1536-41.

52. Li M, Zhuang Y, Shan B. Analysis of expression and functions of histone deacetylase 6 (hdac6). In: Histone deacetylases. Springer; 2016. pp. 85-94.

53. Li Y, Shin D, Kwon SH. Histone deacetylase 6 plays a role as a distinct regulator of diverse cellular processes. FEBS J. 2013;280:775-93.
54. Brush MH, Guardiola A, Connor JH, Yao T-P, Shenolikar S. Deactylase inhibitors disrupt cellular complexes containing protein phosphatases and deacetylases. J Biol Chem. 2004;279:7685-91.

55. Esteves SL, Domingues SC, da Cruz e Silva OA, Fardilha M. da Cruz e Silva EF: Protein phosphatase 1a interacting proteins in the human brain. Omics J Integr Biol. 2012;16:3-17.

56. Beurel E. HDAC6 regulates LPS-tolerance in astrocytes. PLOS ONE. 2011;6:e25804.

57. Fang X, Yu SX, Lu Y, Bast RC, Woodgett JR, Mills GB. Phosphorylation and inactivation of glycogen synthase kinase 3 by protein kinase A. Proc Natl Acad Sci. 2000;97:11960-5.

58. Alao JP, Stavropoulou AV, Lam EW, Coombes RC. Role of glycogen synthase kinase 3 beta (GSK3 3 ) in mediating the cytotoxic effects of the histone deacetylase inhibitor trichostatin A (TSA) in MCF-7 breast cancer cells. Mol Cancer. 2006;5:40.

59. Elie A, Prezel E, Guérin C, Denarier E, Ramirez-Rios S, Serre L, Andrieux A, Fourest-Lieuvin A, Blanchoin L, Arnal I. Tau co-organizes dynamic microtubule and actin networks. Sci Rep. 2015;5:9964.

60. Gimona M, Buccione R, Courtneidge SA, Linder S. Assembly and biological role of podosomes and invadopodia. Curr Opin Cell Biol. 2008;20:235-41.

61. Siddiqui TA, Lively S, Vincent C, Schlichter LC. Regulation of podosome formation, microglial migration and invasion by Ca 2+-signaling molecules expressed in podosomes. J Neuroinflammation. 2012;9:250.

62. Vincent C, Siddiqui TA, Schlichter LC. Podosomes in migrating microglia: components and matrix degradation. J Neuroinflamm. 2012;9:190.

63. Cabrero JR, Serrador JM, Barreiro O, Mittelbrunn M, Naranjo-Suárez S, Martín-Cófreces N, Vicente-Manzanares M, Mazitschek R, Bradner JE, Ávila J. Lymphocyte chemotaxis is regulated by histone deacetylase 6, independently of its deacetylase activity. Mol Biol Cell. 2006;17:3435-45.

64. Gao Y-S, Hubbert CC, Lu J, Lee Y-S, Lee J-Y, Yao T-P. Histone deacetylase 6 regulates growth factor-induced actin remodeling and endocytosis. Mol Cell Biol. 2007;27:8637-47.

65. Biswas S, Kalil K. The microtubule-associated protein tau mediates the organization of microtubules and their dynamic exploration of actinrich lamellipodia and filopodia of cortical growth cones. J Neurosci. 2018;38:291-307.

66. Semenkovich CF, Ostlund RE Jr, Olson MO, Yang JW. A protein partially expressed on the surface of HepG2 cells that binds lipoproteins specifically is nucleolin. Biochemistry. 1990;29:9708-13.

67. Wang S-A, Li H-Y, Hsu T-I, Chen S-H, Wu C-J, Chang W-C, Hung J-J. Heat shock protein 90 stabilizes nucleolin to increase mRNA stability in mitosis. J Biol Chem. 2011;286:43816-29.

\section{Publisher's Note}

Springer Nature remains neutral with regard to jurisdictional claims in published maps and institutional affiliations.
Ready to submit your research? Choose BMC and benefit from:

- fast, convenient online submission

- thorough peer review by experienced researchers in your field

- rapid publication on acceptance

- support for research data, including large and complex data types

- gold Open Access which fosters wider collaboration and increased citations

- maximum visibility for your research: over 100M website views per year

At BMC, research is always in progress.

Learn more biomedcentral.com/submissions 